\title{
Multi-Band Polarization Insensitive Ultra-Thin THz Metamaterial Absorber for Imaging and EMI Shielding Applications
}

\author{
Gaurav Saxena ${ }^{1}$, Yogita Khanna2*, Y K Awasthi ${ }^{3}$, Priyanka Jain ${ }^{4}$ \\ ${ }^{1}$ Electronics \& Communication Engineering, Galgotia College of Engineering and Technology, Greater Noida, India \\ ${ }^{2}$ Electronics \& Communication Engineering, Manav Rachna University, Faridabad HR, India \\ ${ }^{3}$ Electronics \& Communication Engineering, Manav Rachna International Institute of Research and Studies, Faridabad HR, India \\ ${ }^{4}$ Department of Electronics \& Communication Engineering, Delhi Technological University, Delhi, India \\ Corresponding author: Yogita Khanna (e-mail: yogita@mru.edu.in)
}

ABSTRACT In this article, a multi-band polarization-insensitive ultrathin (0.036 $\lambda)$ single layer metamaterial absorber is designed for $\mathrm{THz}$ imaging and EMI shielding. A unique oval-shaped structure is compact with three circular ring-shaped resonators is proposed with a unit cell dimension of $36 \times 36 \times 19.6 \mu \mathrm{m}^{3}$. The absorbance of the proposed multiband MMA is above $90 \%$ for three bands i.e. $98.57 \%, 90 \%$ and $99.85 \%$ at $5.58,7.98-8.84,11.45 \mathrm{THz}$ frequency respectively. Return loss is nearly the same for the changing incident and polarization angle. Therefore, this metamaterial absorber with a wide range of polarization insensitivity is found and it is also suitable for various applications such as quantum RADAR Imaging, energy harvesting, and optoelectronic devices.

INDEX TERMS MMA (Metamaterial Absorber), Triple-band, Polarization-Insensitive, EMC (Electromagnetic Compatibility), Terahertz (THz), EMI (Electromagnetic Interference)

\section{INTRODUCTION}

Tn recent progresses, metamaterials have entered in various 1 practical applications such as sensing, cloaking, stealth or absorber, and communication [1-5]. Among these applications absorbers have been found feasible and applicable in a microwave, terahertz as well as the infrared band for applications like terahertz and infrared imaging, perfect absorbers, wave manipulation, energy harvesting, chemical detection and, radioactive cooling. Moreover, it is also used for single-band, dual-band, multiband and wideband applications. Metamaterials in terahertz range are popular due to bandwidth scalability, high security, and strong penetration $[6-7,17,18]$. Metamaterial absorbers are generally arranged as a threelayered structure comprising of a ground plane as a bottom layer, a dielectric as middle, and a resonant periodic structure as a top layer. In comparison with like Salisbury and Jaumann metamaterial absorbers are an alternative that are used in fifthgeneration fighter plane stealth to improve the isolation of MIMO antenna, imaging, sensing, Radar cross-section and electromagnetic interference reduction [8-9]. Moreover, multiband absorbers that are polarization as well as incident wave insensitive have been designed and validated with a thickness lower than the conventional absorbers having absorptivity above $90 \%$ for $\mathrm{S}$, and $\mathrm{X}$-band applications [10-11].

In this paper, an ultra-thin microwave absorber is designed that works at three frequency bands i.e. band-1(5.42-5.74 THz), band-2 (7.98-8.84THz), and band-3(11.36-11.54THz) with high absorptivity in X-band. The absorption for the three intended bands is above $90 \%$ for TE \& TM polarization. The threelayered structure is simple to design and fabricate using commercially available copper-clad laminate. Section-II shows the design of the proposed MMA structure. Section-III depicts the simulated results of the proposed MMA in terms of return loss, absorbance, reflectivity, current density, RCS, and electric field distribution. Conclusion of the proposed designing work is summarized in section-IV.

\section{DESIGN OF MMA STRUCTURE}

The proposed metamaterial absorber comprises two oval geometries organized as a flower-shaped pattern having three ring-shaped slots cut at the center and a metallic ground plane separated by a dielectric layer, as shown in Figure 1. The MMA is designed on an FR-4 substrate with $19.6 \mu \mathrm{m}$ height (h) and copper plated $\left(\sigma=4.2 \times 10^{7} \mathrm{~S} / \mathrm{m}\right)$ of $3 \mu \mathrm{m}$ thickness $(\mathrm{t})$. FR4 is commercially available and an alternative to the expensive dielectrics with satisfactory response for the $\mathrm{THz}$ range of interest [37-38]. The ground made up of a copper conductor acts as conducting mirror so that there is no transmission of the incident wave and thus converting the incident wave into heat that minimizes the reflected wave which produces high absorbance. The radius and width of all the rings are optimized and the geometrical details of the structure are mentioned in Table-1 and shown in Figure 1.

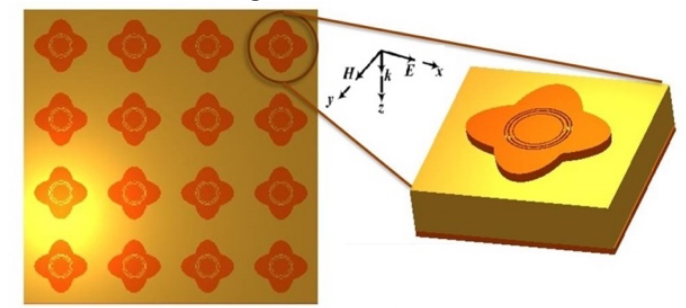

(a)

(b)

FIGURE 1 Triple band meta-material absorber structure (a) Front view (b) Enlarged view

TABLE-I Dimensions of Proposed MMA shown in Figure 2

\begin{tabular}{cccc}
\hline \hline $\begin{array}{c}\mathbf{R} 1 \\
(\boldsymbol{\mu m})\end{array}$ & $\begin{array}{c}\mathbf{R 2} \\
(\boldsymbol{\mu m})\end{array}$ & $\mathbf{R 3}(\boldsymbol{\mu m})$ & $\begin{array}{c}\mathbf{R 4} \\
(\boldsymbol{\mu m})\end{array}$ \\
\hline 8.5 & 10 & 11.3 & 26 \\
\hline \hline $\mathbf{S}$ & $\mathbf{h}$ & $\mathbf{t}$ & \\
$(\boldsymbol{\mu m})$ & $(\boldsymbol{\mu m})$ & $(\boldsymbol{\mu m})$ & \\
\hline 36 & 19.6 & 3 & \\
\hline \hline
\end{tabular}




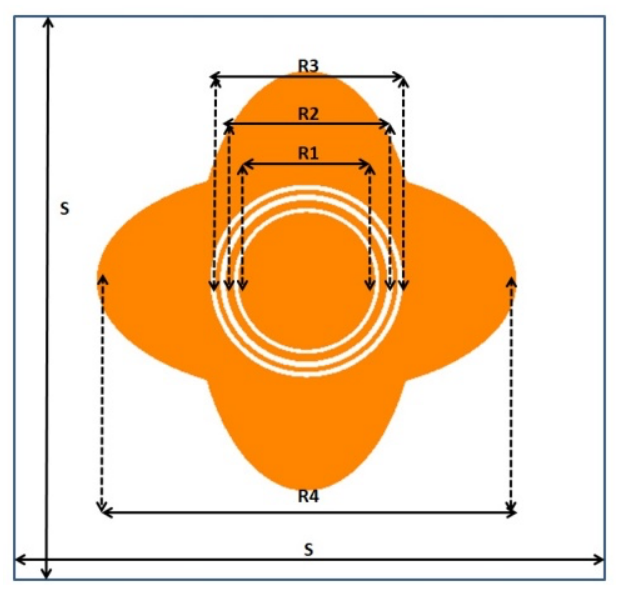

FIGURE 2 Dimensions of a triple-band absorber

Absorption is measured by reflection coefficient $\left(\mathrm{S}_{11}\right)$ and transmission coefficient $\left(\mathrm{S}_{21}\right)$ using the simple relation given in (1) $[21]$ :

$A(\omega)=1-\left|S_{11}\right|^{2}-\left|S_{21}\right|^{2}$

The ground plane is a metallic sheet therefore no transmission takes place, so the transmission coefficient $\left(\mathrm{S}_{21}\right)$ becomes zero. So, (1) is reduced to (2) [21]:

$A(\omega)=1-\left|S_{11}\right|^{2}$

$\left|S_{11}\right|^{2}=\left|S_{11 \text { co }}\right|^{2}-\left|S_{11 \text { cross }}\right|^{2}-\left|S_{21 \text { co }}\right|^{2}-\left|S_{21 \text { cross }}\right|^{2}$

Equation (4) comes from (2) and (3) [21],

$\mathrm{A}(\omega)=1-\left|\mathrm{S}_{11 \text { co }}\right|^{2}-\left|\mathrm{S}_{11 \text { cross }}\right|^{2}-\left|\mathrm{S}_{21 \text { co }}\right|^{2}-\left|\mathrm{S}_{21 \text { cross }}\right|^{2}$ (4)

Where, $S_{11 \text { co and }} S_{11 \text { cross }}$ are the reflection coefficient of copolarized and cross-polarized EM waves as shown in Figure $5(\mathrm{c})$.

The S-parameters are calculated using the simulations done on CST-Microwave Studio for a frequency sweep from $5 \mathrm{THz}$ to $12 \mathrm{THz}$. The absorber impedance should be comparable to the free space impedance to achieve perfect absorption. The normalized impedance can be calculated using (5):

$Z=\sqrt{\frac{\left(1+S_{11}\right)^{2}-S_{21}^{2}}{\left(1-S_{11}\right)^{2}-S_{21}^{2}}}=\sqrt{\frac{\left(1+S_{11}\right)^{2}}{\left(1-S_{11}\right)^{2}}} \quad$ as $S_{21}=0$

Equivalent Circuit Analysis-Transmission line theory is used to explain electromagnetic wave absorbers such as Salisbury, Jaumann, and Dallenbach absorbers, etc. This theory is applicable to $\mathrm{THz}$ metamaterial absorbers because of scalability. Metamaterials using transmission line theory can be modeled as metasurface impedance connected parallel to the dielectric impedance. $Z_{i n} \& Z_{m}$ are the input impedance and impedance of the top layer of the designed metasurface absorber comprising series $R, L \& C$ components. Also, $X_{L} \&$ $X_{C}$ are inductive reactance and capacitive reactance of the equivalent circuit respectively. $Z_{t}$ is the dielectric impedance of the designed absorber by (6) \& (7) [21].

$\mathbf{Z}_{\mathbf{m}}=\mathbf{R}+\mathbf{j}\left(\mathbf{X}_{\mathbf{L}}-\mathbf{X}_{\mathbf{c}}\right)=\mathbf{R}+\mathbf{j}\left(\boldsymbol{\omega} \mathbf{L}-\frac{1}{2 \pi \mathrm{fC}}\right)$

(6)

$\mathrm{Z}_{\mathrm{t}}=\mathbf{j} \frac{\mathrm{Z}_{0}}{\sqrt{\varepsilon_{\mathrm{r}}}} \tan \left(\frac{2 \pi \mathrm{f} \sqrt{\varepsilon_{\mathrm{r}}} \mathbf{h}}{\mathrm{c}}\right)$

(7)

The total impedance of the metasurface absorber $Z_{\text {in }}$ and reflection coefficient is given as:

$\frac{1}{\mathrm{Z}_{\mathrm{in}}}=\frac{1}{\mathrm{z}_{\mathrm{m}}}+\frac{1}{\mathrm{z}_{\mathrm{t}}}$
$\Gamma=\frac{\mathbf{z}_{\text {in }}-\mathbf{z}_{\mathbf{0}}}{\mathbf{z}_{\text {in }}+\mathbf{z}_{\mathbf{0}}}$

Equations (10) and (11) indicate the constraints upon relating $\mathrm{s}$ (6)-(9) in agreement to match with the free space impedance, i.e. $Z_{\text {in }}=Z_{0}$.

$\frac{1}{Z_{0}}=\frac{R}{R^{2}+(2 \pi f L-1 / 2 \pi f C)^{2}}$

$\frac{\sqrt{\varepsilon_{\mathrm{r}}}}{\mathrm{Z}_{0}} \cot \left(\frac{2 \pi \mathrm{f} \sqrt{\varepsilon_{\mathrm{r}}} \mathrm{d}}{\mathrm{c}}\right)=\frac{(2 \pi \mathrm{fL}-1 / 2 \pi \mathrm{fC})}{\mathrm{R}^{2}+(2 \pi \mathrm{fL}-1 / 2 \pi \mathrm{fC})^{2}}$

Put the value of $\frac{\mathbf{1}}{\mathbf{z}_{0}}$ and from (10) in (11) and change "cot" form to "tan" form gives (12) that can be further solved by (13):

$\frac{R \sqrt{\varepsilon_{r}}}{R^{2}+(2 \pi f L-1 / 2 \pi f C)^{2}} \cot \left(\frac{2 \pi f \sqrt{\varepsilon_{r}} d}{c}\right)=\frac{(2 \pi f L-1 / 2 \pi f C)}{R^{2}+(2 \pi f L-1 / 2 \pi f C)^{2}}$

$\frac{R \sqrt{\varepsilon_{r}}}{\tan \left(\frac{2 \pi f \sqrt{\varepsilon_{r}} d}{c}\right)}=(2 \pi f L-1 / 2 \pi f C)$

Putting value of $(2 \pi f L-1 / 2 \pi f C)$ from (11) to (13) gives (14):

$\frac{1}{Z_{0} \tan \left(\frac{2 \pi f \sqrt{\varepsilon_{r}} d}{c}\right)}=\frac{\frac{1}{\tan \left(\frac{2 \pi f \sqrt{\varepsilon_{r}} d}{c}\right)}}{R\left(1+\frac{\varepsilon_{r}}{\tan ^{2}\left(\frac{2 \pi f \sqrt{\varepsilon_{r}} d}{c}\right)}\right)}$

The value of $\mathrm{R}, \mathrm{L}$, and $\mathrm{C}$ can be estimated from (15) and (16) as:

$R=\frac{Z_{0} \tan ^{2}\left(\frac{2 \pi f \sqrt{\varepsilon_{\mathrm{r}}} d}{c}\right)}{\varepsilon_{\mathrm{r}}+\tan ^{2}\left(\frac{2 \pi f \sqrt{\varepsilon_{\mathrm{r}}} \mathrm{d}}{\mathrm{c}}\right)}$

$\frac{\sqrt{\varepsilon_{\mathrm{r}}} \mathrm{Z}_{0} \tan \left(\frac{2 \pi \mathrm{f} \sqrt{\varepsilon_{\mathrm{r}}} \mathrm{d}}{\mathrm{c}}\right)}{\varepsilon_{\mathrm{r}}+\tan ^{2}\left(\frac{2 \pi \mathrm{f} \sqrt{\varepsilon_{\mathrm{r}}} \mathrm{d}}{\mathrm{c}}\right)}=(2 \pi \mathrm{fL}-1 / 2 \pi \mathrm{fC})$

(16)

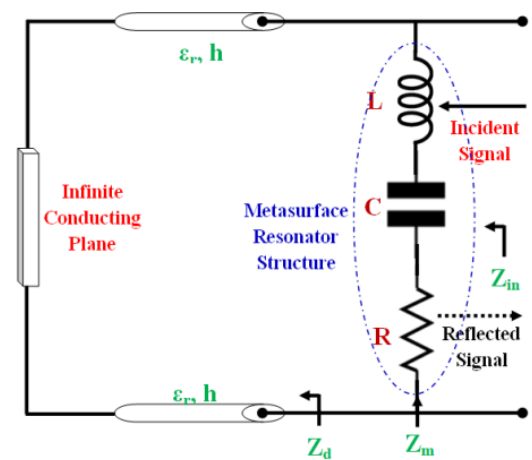

FIGURE 3 Shows the equivalent impedance circuit model of the absorber circuit in terms of series Resistance $(R)$, Inductance $(L)$ \& Capacitance $(C)$

For maximum absorption condition, the impedance of the absorber should be equal to the characteristic impedance of free space as per maximum power transfer theorem i.e. $Z_{i n}=Z_{0}=$ $377 \Omega$ in $[5-9]$. 


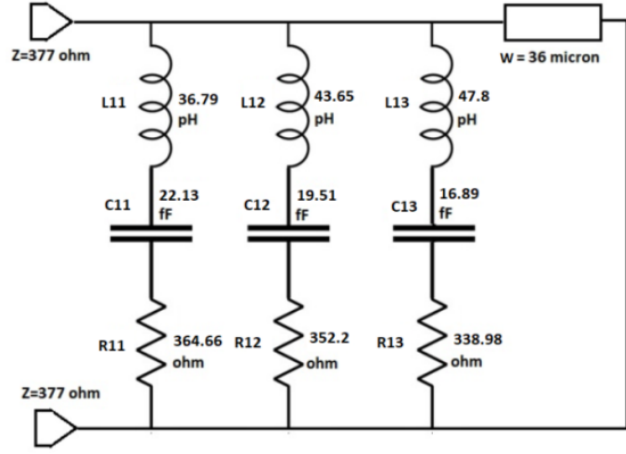

FIGURE 4 Equivalent circuit model of proposed metasurface absorber

Figure4 shows the equivalent circuit diagram of proposed MMA with three distinct resonance frequencies 5.58, 7.98$8.84,11.45 \mathrm{THz}$ having absorbance level more than $90 \%$. For $5.58,7.98-8.84, \quad 11.45 \mathrm{THz}$ resonance frequency with components value are $\mathrm{R}_{11}=364.66 \Omega, \mathrm{R}_{12}=352.2 \Omega, \mathrm{R}_{13}=$ $338.98 \Omega, \mathrm{L}_{11}=36.79 \mathrm{pH}, \mathrm{L}_{12}=43.65 \mathrm{pH}, \mathrm{L}_{13}=47.8 \mathrm{pH}, \mathrm{C}_{11}=$ $22.13 \mathrm{fF}, \mathrm{C}_{12}=19.51 \mathrm{fF}, \mathrm{C}_{13}=16.89 \mathrm{fF}$ respectively.

\section{RESULTS \& DISCUSSION}

The proposed triple-band MMA is simulated with unit cell boundary condition on the $\mathrm{x}-\mathrm{y}$ axis and incident wave on the zaxis to generate a return loss curve as shown in Figure 5 (a). Absorptivity at the intended band $5.58 \mathrm{THz}, 8.71 \mathrm{THz}$, and $11.46 \mathrm{THz}$ is above $90 \%$ and for band-1(5.42-5.74 THz), band-2 $(7.98-8.84 \mathrm{THz})$, and band-3(11.36-11.54THz) having above $90 \%$ as shown in Figure 5 (b). The absorption of the proposed MMA is calculated by (2) [9]. The absorber structure is horizontally and vertically polarized waves to obtain co and cross-reflection coefficients by assigning the Floquet ports on the top of the unit cell as indicated in Fig 5(c). Figure 5(d) shows that the phase change due to resonance, indicating the phase variation of $\pm 180^{\circ}$ at $5.58 \mathrm{THz}, 8.71 \mathrm{THz}$, and $11.46 \mathrm{THz}$. Fig 5(e) indicates the resonance due to the individual ring in the absorber structure at $7.54 \mathrm{THz}, 9.41 \mathrm{THz}$ and $10.418 \mathrm{THz}$ respectively. However, the resonating frequencies of the final structure are difference due to the inter-capacitance between the rings. Fig 5(f) indicates the circuit simulation in comparison to the CST simulation. It shows three resonance frequencies with a small shift.

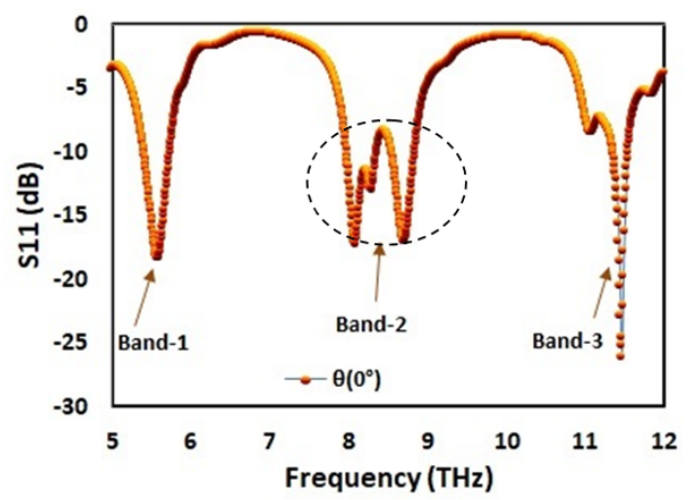

(a)

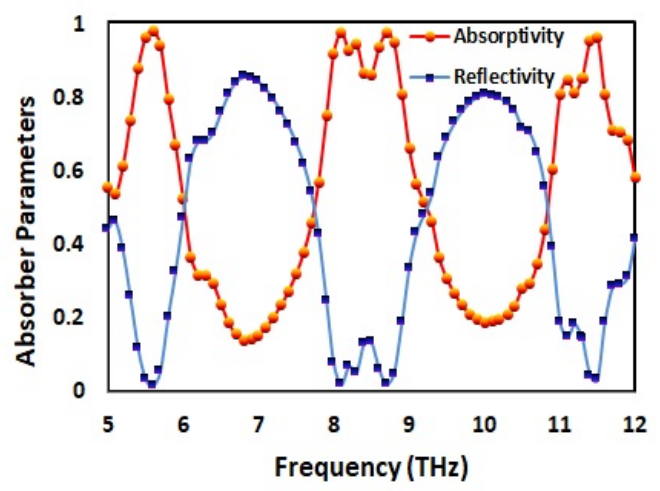

(b)

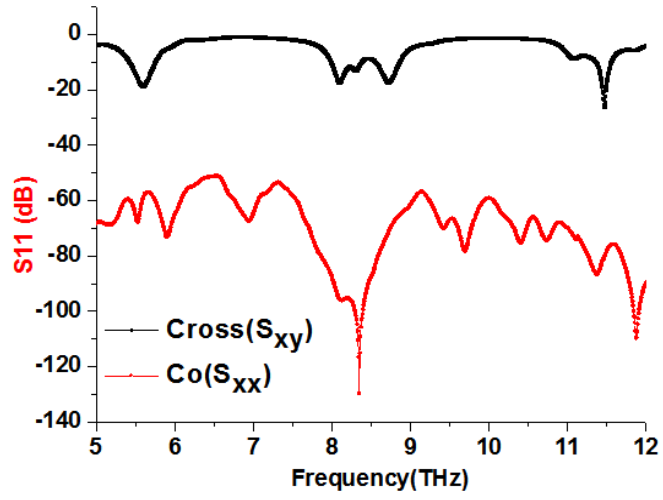

(c)

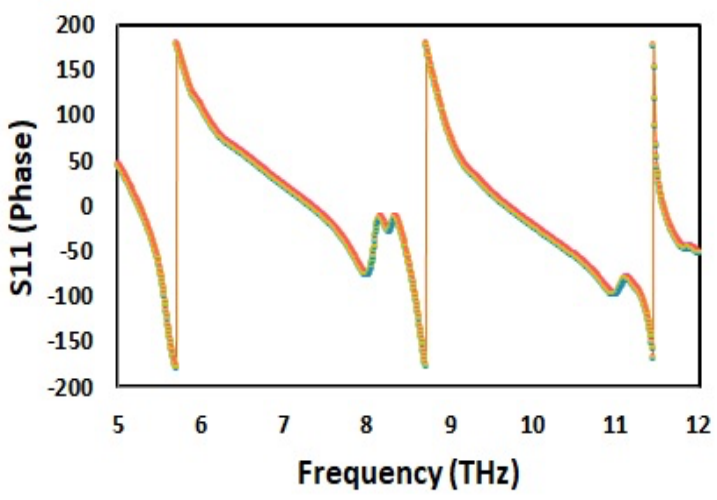

(d)

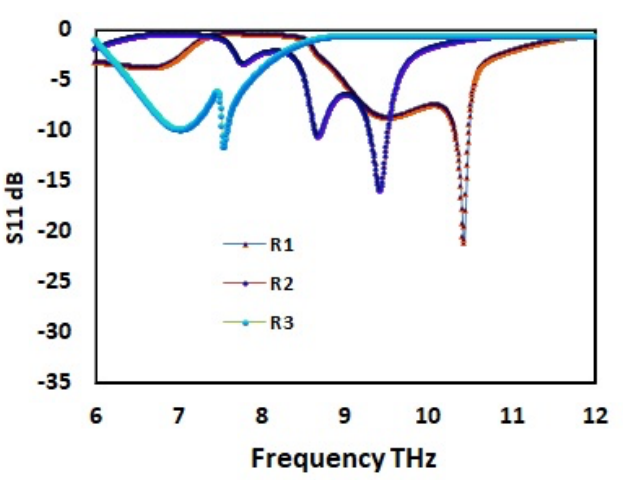

(e) 


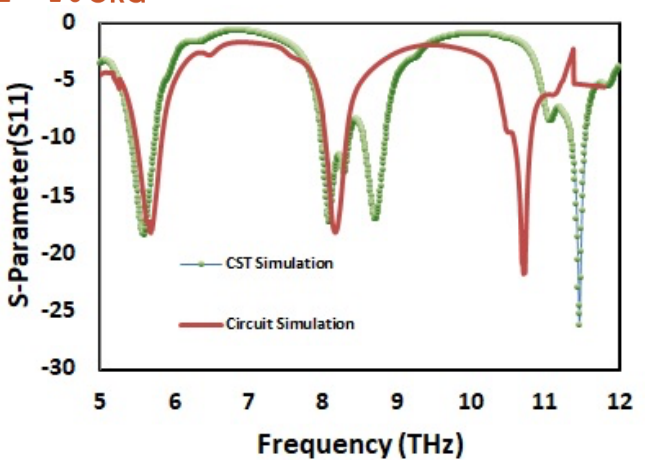

(f)

FIGURE 5 Simulated Results of proposed triple-band absorber (a) Return loss (b) Absorptivity and Reflectivity (c) Co \& Cross $\mathrm{S}_{11}$ (d) Phase shift of proposed Multiband absorber at different frequencies, (e) $S_{11}$ for individual ring structure of Radius R1, R2 \& R3 indicating resonance frequency as $7.54 \mathrm{THz}, 9.41 \mathrm{THz}$ and $10.418 \mathrm{THz}$ respectively, (f) comparison of circuit simulation and CST simulation.

Figure 6 indicates the results of absorbers that are required to recognize the exotic behavior of the material in terms of effective relative permeability/permittivity and refractive index, due to geometrical alterations, not reflected in natural materials. Relative permeability is having a negative peak value, at the resonant frequencies. Figure 6 (a), (b), and (c) show the negative permittivity, permeability, and refractive index at three resonance frequencies respectively. Also, Figure 6 (d) indicates the material (Copper \& FR4) behaviour in the desired frequency band. The single layer absorber can be modelled using Drude's formulation for the metal conductivity as given in (17)[39]:

$\sigma\left(\omega, \mu_{c}, \tau, t\right)=-j \frac{e^{2} k_{B} t}{\pi h^{2}(\omega-j 2 \tau)}\left(\frac{\mu_{c}}{k_{B} t}+2 \ln \left(e^{-\frac{\mu_{c}}{k_{B} t}}+1\right)\right)$

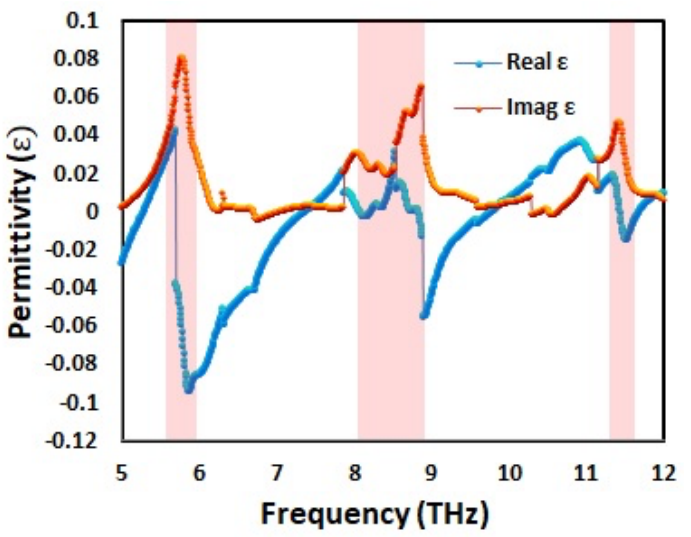

(a)

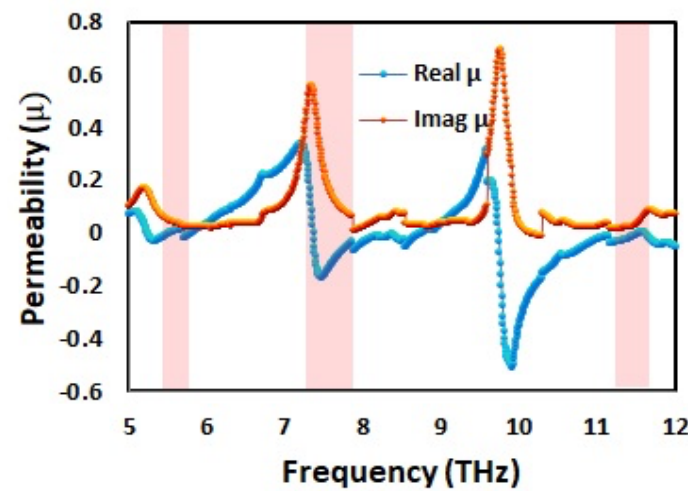

(b)

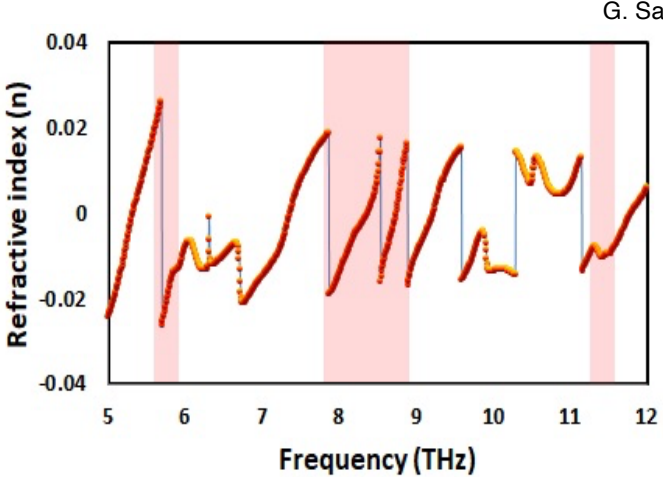

(c)

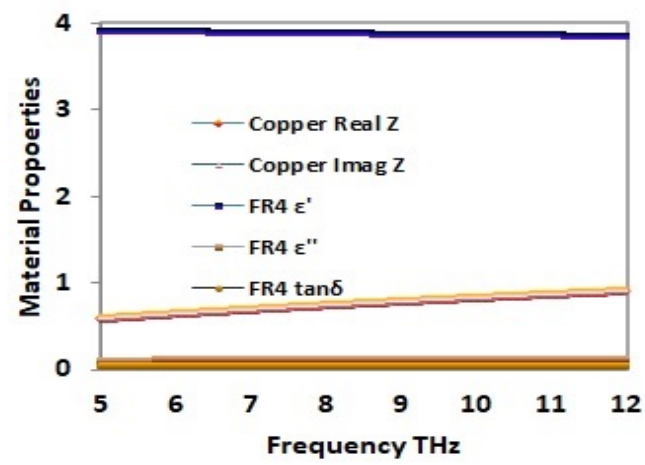

FIGURE 6 Simulated Results concerning the frequency sweep from $5 \mathrm{THz}$ to 13 $\mathrm{THz}$ (a) Permittivity (b) Permeability(c) refractive index of the triple-band absorber (d) Material properties viz. Complex permittivity and Loss Tangent for FR4 and Impedance of copper conductor at the desired frequency band.

where, the parameters $\omega, \mu_{c}, t, \tau, h, e$ and $k_{B}$ are radian frequency, chemical potential, temperature, scattering rate, Plank's constant, electronic charge and Boltzmann's constant.

Figure 7 depicts the performance of the absorber under extreme conditions of orientation and wave incidence. In Figure 7 (a) polarization angle " $\phi$ " is varied from $0^{\circ}$ to $90^{\circ}$ with an incident wave at normal i.e. " $\theta=0^{\circ}$ ". It is observed that the absorber designed is insensitive to the polarization angle. The incidence angle is varied from $0^{\circ}$ to $90^{\circ}$ with " $\phi=0^{\circ}$ ", it is found that the absorber is also insensitive to incidence angle, thus reducing RCS at three frequency bands as shown in Figure 7(b). RCS reduction is used in stealth for fifthgeneration aircraft to minimize the reflected power for detecting the stealth aircraft.

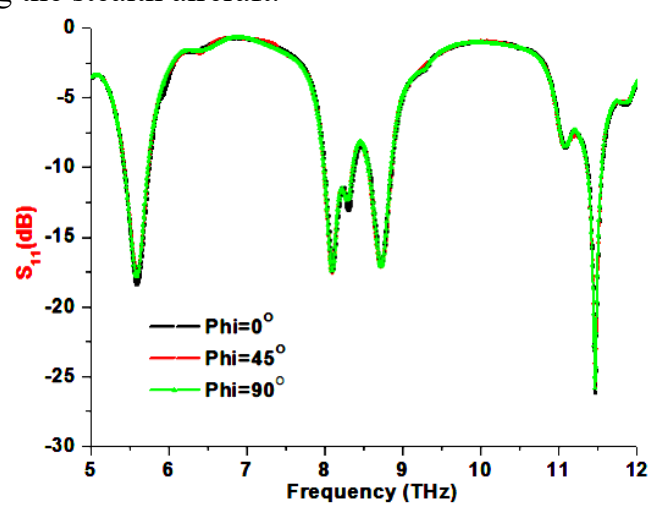

(a) 


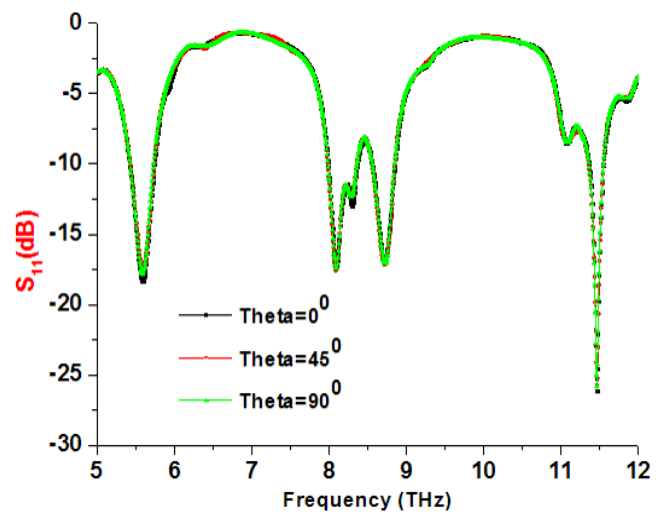

(b)

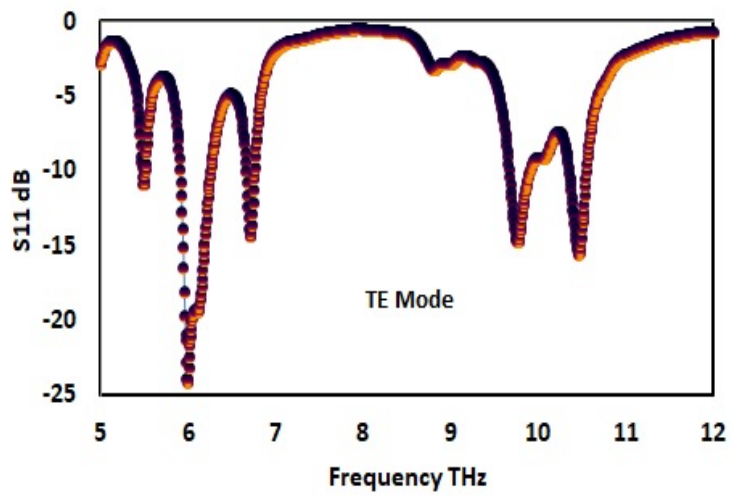

(c)

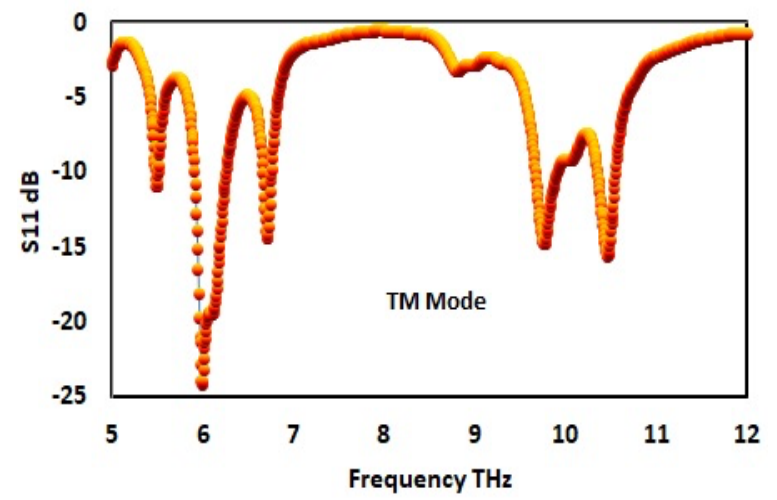

(d)

FIGURE 7 Simulated reflection coefficient " $\mathrm{S}_{11}$ " of the absorber for various (a) polarization angles " $\phi$ " at normal incidence (b) incidence angles " $\theta$ " at " $\phi=0$. "(c)TE polarization, (d) TM polarization

The polarization angle $\phi$, is varied from $0^{\circ}$ to $90^{\circ}$ keeping incident angle $\theta=0^{\circ}$, it can be observed from Figure 7(a)there is no variation in absorptivity, which indicates that the absorber is polarization insensitive. Also, it is clear from Figure 7(b) that if the incident angle $\theta$ is varied from $0^{\circ}$ to $90^{\circ}$, the structure holds good reliability. Therefore, the absorber is insensitive to the angle of incidence of the approaching electromagnetic wave. Fig 7 (c) and (d) indicate the TE and TM polarization respectively.

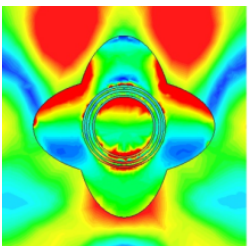

(a)

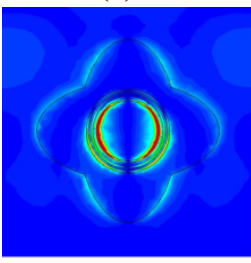

(d)

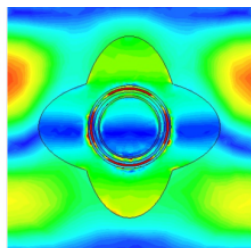

(b)

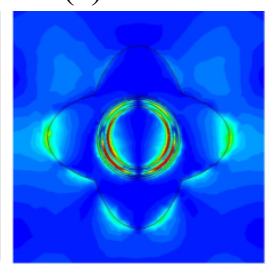

(e)

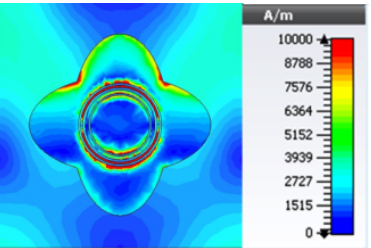

(c)

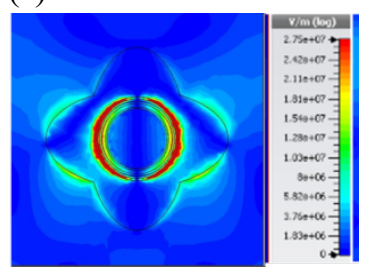

(f)

FIGURE 8 Simulated magnetic field $\mathrm{H}_{\mathrm{z}}$ at (a) $5.58 \mathrm{THz}$ (b) $8.71 \mathrm{THz}$ and (c) 11.46 THz and Electric field $E_{z}$ at (d) $5.58 \mathrm{THz}$, (e) $8.71 \mathrm{THz}$ and (f) $11.46 \mathrm{THz}$ of proposed absorber frequency respectively

It can be stated from the results that the designed absorber is insensitive to incident angle variations as well as polarization angle variations. The absorber structure comprising the ring resonators has peak absorptivity at three bands i.e.5.58THz, $8.71 \mathrm{THz}$, and $11.46 \mathrm{THz}$. Figure 8 shows the structure behavior with the approaching electromagnetic wave indicating the electric and magnetic fields. The simulations are performed using the field monitors in the CST-microwave studio with appropriate periodic boundary conditions (PBC). The top and bottom walls are set with perfect electric conductor (PEC) boundary conditions. Also, perfect magnetic conductor (PMC) boundary conditions are applied to the side walls. The remaining sides are assigned as ports.

Figure8 (a)-(f) shows the magnetic and electric field variations with the operating frequency. Figure9 (a), (b), and (c) show that for lower frequencies the outer structure is responsible for absorption, and at higher frequencies, the electric field is concentrated more on the inner rings. The proposed MMA provides good absorbance and low profile compared with the referred articles as shown in Table-II.

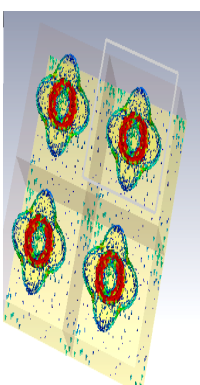

FIGURE 9 Surface current density of proposed absorber at (a) $5.58 \mathrm{THz}$ (b) 8.71THz and (c) $11.46 \mathrm{THz}$ respectively

TABLE-II Comparison table of proposed MMA with existing reference

TABLE-II Comparison table of proposed MMA with existing reference
\begin{tabular}{|c|c|c|c|c|}
\hline Frequency & $\begin{array}{c}\text { Absorbance } \\
\text { Band }\end{array}$ & $\begin{array}{c}\text { Absorban } \\
\text { ce } \\
(\%)\end{array}$ & $\begin{array}{c}\text { Thickness } \\
(\lambda)\end{array}$ & $\begin{array}{c}\text { Polarization } \\
\text { insensitivity/ } \\
\text { Reference }\end{array}$ \\
\hline $0.2-2.4 \mathrm{THz}$ & 2 & $>75$ & $0.13 \lambda$ & Yes[6] \\
\hline $0.1-1 \mathrm{THz}$ & 5 & $>90$ & $0.036 \lambda$ & $\mathrm{No}[13]$ \\
\hline $0-2 \mathrm{THz}$ & 5 & $>94$ & $0.027 \lambda$ & $\mathrm{No}[14]$ \\
\hline $0-4.5 \mathrm{THz}$ & 5 & $>98$ & $1.515 \lambda$ & $\mathrm{Yes}[15]$ \\
\hline
\end{tabular}




\begin{tabular}{|c|c|c|c|c|}
\hline $0.5-4 \mathrm{THz}$ & 4 & $>75$ & $0.13 \lambda$ & Yes[16] \\
\hline $1.9 \& 5 \mathrm{THz}$ & 2 & $>95$ & $0.68 \lambda$ & No[23] \\
\hline $0.1-2 \mathrm{THz}$ & 6 & $>95$ & $0.13 \lambda$ & Yes[25] \\
\hline $1-8 \mathrm{THz}$ & 4 & $>90$ & - & Yes[26] \\
\hline $8-14 \mathrm{GHz}$ & 3 & $>98$ & $0.010 \lambda$ & Yes[27] \\
\hline $2-5 \mathrm{THz}$ & 4 & $>99$ & $0.134 \lambda$ & Yes[28] \\
\hline $4-12 \mathrm{THz}$ & 7 & $>90$ & $0.274 \lambda$ & Yes[29] \\
\hline $6-18 \mathrm{GHz}$ & 5 & $>90$ & - & Yes[35] \\
\hline $85-345 \mathrm{THz}$ & 4 & $>99$ & - & Partial[36] \\
\hline $\begin{array}{c}5.58- \\
11.45 \mathrm{THz} \\
\end{array}$ & 3 & $>91.2$ & $0.013 \lambda$ & Yes [Proposed] \\
\hline
\end{tabular}

[13] He, Y., Wu, Q. \& Yan, S. "Multi-Band Terahertz Absorber at 0.1-1 $\mathrm{THz}$ Frequency Based on Ultra-Thin Metamaterial", Plasmonics, vol. 14, pp. 1303-1310, 2019.

[14] Sabah, C., Mulla, B., Altan, H. et al. "Cross-like terahertz metamaterial absorber for sensing applications", Pramana - J Phys vol. 91, pp.17-23, 2018

[15] Tianhua Meng, Dan Hu, Qiaofen Zhu, "Design of a five-band terahertz perfect metamaterial absorber using two resonators", Optics Communications, vol. 415, pp. 151-155, 2018.

[16] Yang Liu, Sheng-Qiu Xu, Ming Liu, Xin-Guang Hu, Yong-Fa Duan, Lin Yi, "Tunable multiband terahertz absorber based on a one-dimensional heterostructure containing semiconductor", Optik, vol. 170, pp. 203-209, 2018.

[17] Saxena, Gaurav, Y. K. Awasthi, and Priyanka Jain. "High Isolation and High Gain Super-Wideband (0.33-10 THz) MIMO Antenna for THz Applications." Optik, vol. 223, pp. 165335, 2020.

[18] Khanna, Y., Awasthi, Y.K. Ultra-Thin Wideband PolarizationInsensitive Metasurface Absorber for Aviation Technology. Journal of Electronic Materials, vol. 49, pp. 6410-6416, 2020.

IV. CONCLUSION

A single layer ultra-thin multi-band $\mathrm{THz}$ MMA with polarization-insensitive has been presented with high absorbance at three distinct frequencies 5.58, 8.71, and 11.45THz. The proposed structure has high absorbance with ultra-thin $0.013 \lambda$ thickness with existing literature used in Table-II. So this MMA is used in many defense applications like stealth Fifth-generation stealth aircraft for RCS reduction, MIMO antenna isolation improvement, EMI shielding, energy harvesting, EMC reduction, $\mathrm{THz}$ imaging [18], optoelectronic devices, and sensing in the intended band.

\section{REFERENCES}

[1] Hui Feng Ma, Sanming Hu, Yun Gui Ma, Yun Lai, and Karu Esselle, "The Applications of Metamaterials", International Journal of Antennas and Propagation, vol. 20, pp.1-2, (2015).

[2] Mohammed R Hashemi, Semih Cakmakyapan, and Mona Jarrahi, "Reconfigurable metamaterials for terahertz wave manipulation", Reports on Progress in Physics vol. 80, pp. 1-42 (2017).

[3] Y. Khanna and Y. K. Awasthi, "Wideband Ultra-thin Metamaterial Absorber for Ku \& K- Band Applications," 2020 7th International Conference on Signal Processing and Integrated Networks (SPIN), Noida, India, 2020.

[4] Saxena, Gaurav, Y. K. Awasthi, and Priyanka Jain. "Design of Metasurface Absorber for Low RCS and High Isolation MIMO Antenna for Radio Location \& Navigation." AEU-International Journal of Electronics and Communications, vol. 20, pp. 311712(2021)

[5] R. Xing, C. Dong, B. Zhu, Y. Gao, and S. Jian, "Numerical Analysis on Ribbon-ArraySheet Coupled Graphene Terahertz Absorber," in IEEE Photonics Technology Letters, vol. 28, pp. 2207-2210, 2016.

[6] Y. Wang et al., "Properties and Sensing Performance of AllDielectric Metasurface THz Absorbers," in IEEE Transactions on Terahertz Science and Technology, vol. 10, pp. 599-605, 2020.

[7] R. Mishra, R. Panwar and D. Singh, "Equivalent Circuit Model for the Design of Frequency-Selective, Terahertz-Band, GrapheneBased Metamaterial Absorbers," in IEEE Magnetics Letters, vol. 9, pp. 1-5, 2018.

[8] Y. Wang et al., "Properties and Sensing Performance of AllDielectric Metasurface THz Absorbers," in IEEE Transactions on Terahertz Science and Technology, vol. 10, pp. 599-605, 2020.

[9] M. Qu, T. Chang, G. Guo, and S. Li, "Design of Graphene-Based Dual-Polarized Switchable Rasorber/Absorber at Terahertz," IEEE Access, vol. 8, pp. 127220- 127225, 2020.

[10] M. Biabanifard, A. Arsanjani, M. S. Abrishamian, and D. Abbott, "Tunable Terahertz Graphene-Based Absorber Design Method Based on a Circuit Model Approach," IEEE Access, vol. 8, pp. 70343-70354, 2020

[11] Jin Huang, Jining Li, Yue Yang, Jie Li, Jiahui Li, Yating Zhang, and Jianquan Yao, "Broadband terahertz absorber with a flexible, reconfigurable performance based on hybrid-patterned vanadium dioxide metasurfaces," Optics Express vol. 28, pp. 17832-17840, 2020 .

[12] A. Chen and Z. Song, "Tunable Isotropic Absorber With Phase Change Material VO2," in IEEE Transactions on Nanotechnology, vol. 19, pp. 197-200, 2020.

19] Y. Dong, P. Liu, D. Yu, G. Li, and L. Yang, "A Tunable Ultrabroadband Ultrathin Terahertz Absorber Using Graphene Stacks," in IEEE Antennas and Wireless Propagation Letters, vol. 16, pp. 1115 $1118,2017$.

[20] W. Pan, X. Yu, J. Zhang, and W. Zeng, "A Novel Design of Broadband Terahertz Metamaterial Absorber Based on Nested Circle Rings," in IEEE Photonics Technology Letters, vol. 28, pp. 2335-2338, 2016

[21] Saxena, Gaurav, R. L. Yadava, Priyanka Jain, and Y. K. Awasthi. "Triple Band Polarization Insensitive Ultra-Thin Metamaterial Absorber for EMC and RCS Reduction in X-Band Applications." In IEEE, 2020 7th International Conference on Signal Processing and Integrated Networks (SPIN), pp. 772-775. 2020.

[22] Khan, B., Kamal, B., Ullah, S. et al. Design and experimental analysis of dual-band polarization converting metasurface for microwave applications. Scientific Reports vol. 10, pp.15393, 2020.

[23] Barzegar-Parizi, Saeedeh, Amir Ebrahimi, and Kamran Ghorbani. "High-Q dual-band graphene absorbers by selective excitation of graphene plasmon polaritons: Circuit model analysis." Optics \& Laser Technology 132 (2020): 106483.

[24] Yan, Fei, Li Li, Ruoxing Wang, Hao Tian, Jianlong Liu, Jianqiang Liu, Fengjun Tian, and Jianzhong Zhang. "Ultrasensitive tunable terahertz sensor with graphene plasmonic grating." Journal of Lightwave Technology vol. 37, pp. 1103-1112, 2019.

[25] Biabanifard, Mohammad, and Mohammad Sadegh Abrishamian. "Multi-band circuit model of tunable $\mathrm{THz}$ absorber based on graphene sheet and ribbons." AEU-International Journal of Electronics and Communications vol. 95 pp. 256-263, 2018.

[26] Barzegar-Parizi, Saeedeh, and Amir Ebrahimi. "Ultrathin, polarization-insensitive multi-band absorbers based on graphene metasurface with $\mathrm{THz}$ sensing application." JOSA B vol. 37, pp. 2372-2381,2020.

[27] Wu, Jun. "Tunable multi-band terahertz absorber based on graphene nano-ribbon metamaterial." Physics Letters A vol. 383, pp. 25892593, 2019

[28] Zamzam, Pouria, Pejman Rezaei, and Seyed Amin Khatami. "Quadband polarization-insensitive metamaterial perfect absorber based on bilayer graphene metasurface." Physica E: Low-dimensional Systems and Nanostructures vol. 128, pp. 114621, 2021.

[29] Cheng, Y., Zou, Y., Luo, H. et al. Compact Ultra-Thin Seven-Band Microwave Metamaterial Absorber Based on a Single Resonator Structure. Journal of Elec Materi vol. 48, pp. s3939-3946, 2019.

[30] Wang, Yingying, Zeqiang Chen, Danyang Xu, Zao Yi, Xifang Chen, Jian Chen, Yongjian Tang, Pinghui Wu, Gongfa Li, and Yougen Yi. "Triple-band perfect metamaterial absorber with good operating angle polarization tolerance based on split ring arrays." Results in Physics vol. 16, pp. 102951, 2020.

[31] Amiri, Majid, Farzad Tofigh, Negin Shariati, Justin Lipman, and Mehran Abolhasan. "Wide-angle metamaterial absorber with highly insensitive absorption for TE and TM modes." Scientific reports vol. 10, pp. 1-13, 2020.

[32] Ghods, Mohammad Mahdi, and Pejman Rezaei. "Ultra-wideband microwave absorber based on uncharged graphene layers." Journal of Electromagnetic Waves and Applications vol. 32, pp. 1950-1960, 2018

[33] Yi, Zao, Hang Lin, Gao Niu, Xifang Chen, Zigang Zhou, Xin Ye, Tao Duan, Yong Yi, Yongjian Tang, and Yougen Yi. "Graphenebased tunable triple-band plasmonic perfect metamaterial absorber with good angle-polarization-tolerance." Results in Physics vol. 13, pp. 102149, 2019. 
[34] Yongzhi Chenga, Hui Luo, and Fu Chen, "Broadband metamaterial microwave absorber based on asymmetric sectional resonator structures", Journal of Applied Physics vol. 127, pp. 214902, 2020.

[35] Fen Zhang, Chao Jiang, Qiang Wang, Zixiang Zhao, Yan Wang, Zuojuan Du, Chunqi Wang and Xiaozhong Huang, "A multi-band closed-cell metamaterial absorber based on a low-permittivity alldielectric structure", Applied Physics Express, vol. 13, pp. 084001, 2020.

[36] Hongsen Zhang, Yongzhi Cheng, Fu Chen, "Quad-band plasmonic perfect absorber using all-metal nanostructure metasurface for refractive index sensing", Optik, vol. 229 pp. 166300, 2021.

[37] Limie Qi and Chang Liu, "Terahertz wide angle metamaterial absorber fabricated by printed circuit bosrd technique", Journal of Applied Physics, vol. 124, pp. 153101, 2018.

[38] Ben-Xin Wang, Yuanhao He, Pengcheng Lou and Wenhui Xing, "Design of a dual-band terahertz metamaterial absorber using two identical square patches for sensing application" Nanoscale Advances, vol. 2, pp 763-769, 2020.

[39] Gaurav Varshney, "Reconfigurable Graphene Antenna for $\mathrm{THz}$ Applications: A Mode Conversion Approach" Nanotechnology, vol. 31, pp. 135208, 2020. 